\title{
EFEITO DA HOMEOPATIA Ammonium Carbonicum NA MINIMIZAÇÃO DA LIXIVIAÇÃO DE NITRATO ${ }^{1}$
}

\author{
Effect of the homeopathy Ammonium Carbonicum in the minimization of nitrate leaching
}

\author{
Carolina Cardoso Lisboa ${ }^{2}$, Sérgio Ely Valadão Gigante de Andrade Costa ${ }^{3}$, \\ Daniel Melo de Castro ${ }^{4}$, João José Marques ${ }^{5}$
}

\begin{abstract}
RESUMO
Avaliou-se o efeito da solução homeopática Ammonium Carbonicum em duas dinamizações, $\mathrm{CH} 3$ e CH30, sobre a lixiviação de $\mathrm{NO}_{3}^{-}$. A hipótese inicial foi a de que essas soluções homeopáticas atuariam na microbiota do solo otimizando a utilização de $\mathrm{N}$ pela planta. Esse estudo foi conduzido em casa-de-vegetação e utilizaram-se amostras deformadas do horizonte A de um Latossolo Vermelho distroférrico típico muito argiloso, $0-0,15 \mathrm{~m}$, localizado no campus da UFLA. As colunas de solo foram semeadas com milho (Zea mays L.) e tinham 0,20 x 0,20 m. A irrigação e medição do volume lixiviado foi realizada diariamente. A aplicação dos tratamentos homeopáticos foi realizada via irrigação. As análises do lixiviado foram feitas duas vezes por semana. No lixiviado, mediram-se os teores de $\mathrm{NO}_{3}^{-}$e $\mathrm{NH}_{4}^{+}$. Após 93 dias, as colunas foram seccionadas em camadas de $0,05 \mathrm{~m}$ e as amostras, analisadas quanto à fertilidade, incluindo $\mathrm{NO}_{3}^{-}, \mathrm{NH}_{4}^{+} \mathrm{e} \mathrm{N}$ total. $\mathrm{O}$ delineamento experimental foi inteiramente casualizado, com três repetições, num esquema duplo-cego com placebo. O efeito da solução homeopática na minimização da lixiviação de $\mathrm{NO}_{3}^{-}$não foi relevante nas condições deste estudo.
\end{abstract}

Termos para indexação: Ammonium Carbonicum, agricultura orgânica, nitrato.

\section{ABSTRACT}

The objective of this study was to evaluate the effect of the homeopathic solution Ammonium Carbonicum in two dynamizations $\mathrm{CH} 3$ and $\mathrm{CH} 30$ on $\mathrm{NO}_{3}^{-}$leaching. The early hypothesis was that those homeopathic solutions would act upon soil microbiota optimizing the use of $\mathrm{N}$ by the plant. This study was conducted in greenhouse and samples of the A horizon of a very clayey typic dystroferric Red Latosol, $0-0.15 \mathrm{~m}$, situated on the UFLA campus were utilized. The soil columns were sowed with corn (Zea mays L.) and were $0.20 \times 0.20 \mathrm{~m}$. Irrigation and leached volume measurement was performed daily. The application of the homeopathic treatments was accomplished via irrigation. The analyses of the leachate were done twice a week. In the leachate, the contents of $\mathrm{NO}_{3}^{-}$and $\mathrm{NH}_{4}^{+}$were measured. After 93 days, the columns were sectioned in 0.05 -m layers and the samples were analyzed in relation to fertility, including $\mathrm{NO}_{3}^{-}, \mathrm{NH}_{4}^{+}$and total $\mathrm{N}$. The experimental design was completely randomized with three replicates, in a double-blind scheme with a placebo. The effect of the homeopathic solution in minimizing $\mathrm{NO}_{3}^{-}$leaching was not significant in the conditions of this study.

Index terms: Ammonium Carbonicum, organic agriculture, nitrate.

\section{(Recebido em 9 de agosto de 2004 e aprovado em 27 de março de 2006)}

\section{INTRODUÇÃO}

O crescimento da agricultura orgânica tem despertado a necessidade de se desenvolver técnicas para melhor gerenciar este sistema de produção agrícola, tornando-o mais eficiente, sobretudo no manejo dos nutrientes essenciais. Neste contexto, os adubos orgânicos, de origem animal ou vegetal, e o $\mathrm{N}$ presente na matéria orgânica do solo destacam-se como fontes fornecedoras de $\mathrm{N}$ às plantas.

A restrição ao uso de fertilizantes nitrogenados sintéticos na agricultura orgânica deve-se, principalmente, à sua alta solubilidade. Em função desta alta solubilidade, tem-se atribuído à utilização de fertilizantes nitrogenados sintéticos nos sistemas agrícolas convencionais a degradação da qualidade de águas super e subsuperficiais, sobretudo devido ao nitrato, presente nos adubos ou convertido pela oxidação do $\mathrm{NH}_{4}^{+}$. $\mathrm{O}$ íon $\mathrm{NO}_{3}^{-}$no sistema solo-água apresenta grande mobilidade, o que o torna susceptível a perdas por lixiviação (ELMI et al., 2002; PANG \& LETEY, 2000).

A homeopatia insere-se como uma alternativa na otimização da utilização dos recursos naturais pelas plantas, além de possibilitar outros benefícios à natureza por ser ecológica, sistêmica e de baixo custo (ANDRADE et al., 2001). A homeopatia está oficializada como insumo permitido na agropecuária orgânica (BRASIL, 1999),

${ }^{1}$ Parte da dissertação de mestrado do primeiro autor apresentada à Universidade Federal de Lavras/UFLA - Departamento de Ciência do Solo. ²Engenheira Agrônoma, MS, Departamento de Ciência do Solo/DCS - Universidade Federal de Lavras/UFLA - Cx. P. 3037 - $37200-000$ - Lavras, MG ${ }^{3}$ Acadêmico do Curso de Agronomia, bolsista PIBIC/CNPq. Departamento de Ciência do Solo/DCS - Universidade Federal de Lavras/UFLA Cx.P. 3037 - 37200-000 - Lavras, MG.

${ }^{4}$ Professor, Departamento de Biologia/DBI - Universidade Federal de Lavras/UFLA - Cx. P. 3037 - 37200-000 - Lavras, MG

${ }_{5}^{5}$ Professor, Departamento de Ciência do Solo/DCS - Universidade Federal de Lavras/UFLA - Cx. P. 3037 - $37200-000$ - Lavras, MG - jmarques@ufla.br 
apresentando-se como um recurso coerente com este sistema de produção.

A hipótese do mecanismo de ação das soluções homeopáticas baseia-se na idéia de que a água retém algum tipo de "memória" dos sais que nela foram dissolvidos. Tal evidência foi sugerida por Rey (2003) que, ao utilizar a técnica da termoluminescência para estudar as ligações entre moléculas de água pura e compará-las às ligações entre as moléculas de uma solução altamente diluída, observou que, nas soluções altamente diluídas, a conformação das ligações entre as moléculas de água mostrou-se diferente daquela observada nas moléculas de água pura. Provavelmente, esta mudança estrutural pode ser atribuída às sucessivas e vigorosas agitações que ocorrem durante a dinamização, processo utilizado no preparo de soluções homeopáticas.

No preparo da solução homeopática, por convenção, é utilizada a escala centesimal clássica, em que, cada diluição, guarda a proporção de 1:100 (soluto : solvente). A partir da $12^{\text {a }}$ diluição sucessiva na escala centesimal $\left(100^{12}=10^{24}\right)$ ultrapassa-se o número de Avogrado $\left(6,023 \times 10^{23}\right)$, não havendo mais, para qualquer finalidade prática, presença de moléculas ou íons da substância original (ANDRADE et al., 2001; CARVALHO et al., 2004).

Idealmente, experimentos visando testar a eficiência de um dado medicamento, homeopático ou não, devem ser realizados sob um esquema "duplo-cego", em que o experimentador e o aplicador não saibam qual é o medicamento em teste, controlando, assim, o efeito placebo (CARLINI et al., 1987; citados por CARVALHO et al., 2004).

Agricultores de vários locais, no Brasil e também em outros países como Inglaterra, Cuba e Itália, vêm utilizando a homeopatia em plantas com resultados aparentemente positivos no aumento da resistência a parasitas e doenças, melhoria de condições debilitadas, florescimento, quebra de dormência de sementes e produção de mudas sadias (ANDRADE et al., 2001; CARVALHO et al., 2003, 2004; CASTRO, 2002; HAMMAN et al., 2003). Pesquisas científicas comprovando os resultados obtidos são ainda incipientes nesta área (BETTI et al., 2003). Entretanto, a maior parte desses trabalhos tem sido realizada aplicando homeopatia às plantas. Não há relatos científicos acerca da utilização de homeopatia diretamente aplicada a solos ou à sua microbiota.

Este estudo teve como objetivo verificar a eficiência e os efeitos de duas dinamizações homeopáticas de Ammonium Carbonicum na redução da lixiviação de $\mathrm{NO}_{3}^{-}$. A hipótese inicial deste trabalho é a ação positiva dos princípios homeopáticos sobre a microbiota do solo, otimizando o aproveitamento de $\mathrm{N}$ pelas plantas e, consequientemente, minimização da lixiviação de $\mathrm{NO}_{3}^{-}$.

\section{MATERIAL E MÉTODOS}

O presente estudo foi conduzido em casa-devegetação do Departamento de Ciência do Solo da Universidade Federal de Lavras. Utilizaram-se colunas de solo com amostras deformadas $(<4 \mathrm{~mm})$ acondicionadas em tubos de PVC. Os tubos de PVC tinham 0,20 x 0,20 m. Na parte inferior dos tubos, foi colocada lã-de-vidro como meio filtrante seguida por uma placa de isopor perfurada $(0,05 \mathrm{~m}$ de espessura) para facilitar a percolação do lixiviado. Utilizou-se um funil de plástico de $0,20 \mathrm{~m}$ de diâmetro para conduzir o lixiviado a um recipiente coletor. $\mathrm{Na}$ parte superior das colunas foi inserida no solo, a $30 \mathrm{~mm}$ de profundidade, uma secção de tubo de PVC com 0,15 x 0,10 m. Adotou-se este procedimento para evitar que ocorresse fluxo preferencial da água de irrigação junto às paredes dos tubos de PVC e, deste modo, logrou-se obter um maior volume molhado de solo via irrigação. Cada tubo de PVC foi preenchido com 4,8 $\mathrm{kg}$ de solo, em base seca, perfazendo um volume de 4,24 L.

Utilizou-se o horizonte A de um Latossolo Vermelho distroférrico típico textura muito argilosa, localizado no Sul de Minas Gerais, o qual estava sob pastagem. A profundidade de coleta foi de $0-0,15 \mathrm{~m}$. Este solo apresenta 690, 160 e $150 \mathrm{~g} \mathrm{~kg}^{-1}$, de argila, silte e areia. Calculou-se a necessidade de calagem pelo método da saturação por bases (ALVAREZ \& RIBEIRO, 1999), para elevá-la a $60 \%$. A dose calculada foi $1 \mathrm{t} \mathrm{ha}^{-1}(2,4 \mathrm{~g}$ de calcário por tubo de $\mathrm{PVC}$ ). Aplicou-se calcário magnesiano, com $12 \%$ de $\mathrm{MgO}$, $42 \%$ de $\mathrm{CaO}$ e PRNT $97 \%$, cerca de 50 dias antes da montagem do experimento. Características do solo antes e após calagem estão apresentadas na Tabela 1 .

No dia da montagem das colunas de solo, foi aplicada dose correspondente a $14,11 \mathrm{mg}$ de $\mathrm{H}_{3} \mathrm{BO}_{3}$ por coluna de solo $\left(\sim 1 \mathrm{~kg} \mathrm{ha}^{-1}\right)$. Paralelamente, adicionou-se $1.875 \mathrm{~kg} \mathrm{ha}^{-1}$ de superfosfato simples e $626 \mathrm{~kg} \mathrm{ha}^{-1}$ de sulfato de potássio, adubos permitidos na agricultura orgânica de acordo com a Instrução Normativa $n^{\circ} 7$ (BRASIL, 1999), a fim de obter uma dose de aplicação correspondente a $300 \mathrm{~kg} \mathrm{ha}^{-1}$ de $\mathrm{K}_{2} \mathrm{O}$ e $\mathrm{P}_{2} \mathrm{O}_{5}$. Esses foram inteiramente misturados ao solo.

As colunas de solo foram semeadas com milho (Zea mays L., híbrido simples experimental desenvolvido no DAG-UFLA). Cada coluna foi semeada com três sementes e realizou-se o desbaste de duas plantas 20 dias após o plantio, visando conduzir apenas uma planta por tubo de PVC. As plantas de milho foram cultivadas durante 63 dias. No fim do experimento, elas foram cortadas rente ao solo e encaminhadas para análise da parte aérea. 
TABELA 1 - Características do solo antes e após calagem e adubação. Análises efetuadas conforme Embrapa (1997) e Silva (1999).

\begin{tabular}{lcc}
\hline \multicolumn{1}{c}{ Características } & Antes da calagem & Após a calagem \\
\hline $\mathrm{pH}\left(\mathrm{H}_{2} \mathrm{O}\right)$ & 5,6 & 6,3 \\
$\mathrm{P}\left(\mathrm{mg} \mathrm{kg}^{-1}\right)$ & 6,2 & 7,5 \\
$\mathrm{~K}\left(\mathrm{mg} \mathrm{kg}^{-1}\right)$ & 36 & 41 \\
$\mathrm{Ca}\left(\mathrm{cmol}_{\left.\mathrm{c} ~ \mathrm{~g}^{-1}\right)}\right.$ & 3,5 & 4,1 \\
$\mathrm{Mg}\left(\mathrm{cmol}_{\mathrm{c}} \mathrm{kg}^{-1}\right)$ & 0,6 & 0,8 \\
$\mathrm{C}$ orgânico $\left(\mathrm{g} \mathrm{kg}^{-1}\right)$ & 14,5 & 14,5 \\
$\mathrm{Saturação} \mathrm{por} \mathrm{bases}(\%)$ & 57 & 63 \\
$\mathrm{~N}$ total $\left(\mathrm{g} \mathrm{kg}^{-1}\right)^{*}$ & 3 & 2 \\
$\mathrm{NO}_{3}^{-}\left(\mathrm{mg} \mathrm{kg}^{-1}\right)$ & 103 & 74 \\
$\mathrm{NH}_{4}^{+}\left(\mathrm{mg} \mathrm{kg}^{-1}\right)$ & 22 & 26 \\
\hline
\end{tabular}

*N-total = N-orgânico $+\mathrm{N}$-mineral $\left(\mathrm{NO}_{3}^{-}+\mathrm{NH}_{4}^{+}\right)$.

Este experimento teve duração de 93 dias e ao término desse período realizou-se a amostragem da coluna de solo para submetê-la a análises de fertilidade do solo, conforme os métodos de Embrapa (1997) e Silva (1999). As amostras foram obtidas das profundidades: $0-0,05$; 0,05-0,10; e 0,10-0,15 m. A irrigação do experimento foi realizada com água destilada sobre cada coluna de lixiviação, totalizando $17 \mathrm{~L}$, correspondente a $500 \mathrm{~mm}$ de precipitação.

$\mathrm{O}$ delineamento experimental foi inteiramente casualizado com três repetições. O experimento constou de três tratamentos: H1 - homeopatia Ammonium Carbonicum (CH3); H2 - homeopatia Ammonium Carbonicum (CH30); TA - testemunha sem homeopatia, tratada com água destilada. O experimento foi conduzido num esquema "duplo-cego".

Os compostos homeopáticos foram preparados a partir de $\left(\mathrm{NH}_{4}\right)_{2} \mathrm{CO}_{3}$, conforme Lathoud (2002), em duas dinamizações, $\mathrm{CH} 3$ e $\mathrm{CH} 30$, as quais correspondem à diluição de $1 \mathrm{~g}$ de $\left(\mathrm{NH}_{4}\right)_{2} \mathrm{CO}_{3} 10^{6}$ e $10^{60}$ vezes. Os tratamentos homeopáticos foram aplicados via irrigação, aplicando-se uma gota $(\sim 0,1 \mathrm{~mL})$ do composto homeopático ou do placebo a cada $100 \mathrm{~mL}$ de água destilada. Com tais diluições, pode-se afirmar que nenhum dos tratamentos recebeu adubação nitrogenada.

$\mathrm{O}$ volume lixiviado foi coletado diariamente e medido. $\mathrm{O}$ volume em $\mathrm{mL}$ obtido em cada coluna foi multiplicado pelo valor de 0,472 para expressar o volume lixiviado em $\mathrm{m}^{3} \mathrm{ha}^{-1}$. Os resultados de volume percolado também estão expressos na unidade "Volume de Poros", que é a forma padrão para estudos de lixiviação em solos. A quantidade de água correspondente a 1,0 volume de poros é igual à quantidade de água suficiente para ocupar todo o espaço poroso do solo. Portanto, uma coluna que tenha sido percolada com 2,0 volumes de poros já foi lixiviada com água correspondente a duas vezes seu espaço poroso (isto é: já foi "lavada" duas vezes). A expressão volume de poros permite comparar resultados de solos com várias porosidades e estudados em colunas de tamanhos diferentes.

A análise química da solução lixiviada foi realizada em apenas duas das sete coletas semanais, distanciadas de 3 a 4 dias. Os teores de $\mathrm{NO}_{3}^{-}$e $\mathrm{NH}_{4}^{+}$foram obtidos pelo método padrão destilação-titulação (BREMNER, 1965).

Na parte aérea da planta, quantificaram-se as massas frescas e secas. Posteriormente, o material seco foi triturado em moinho. As análises das amostras de solo e das plantas foram feitas de acordo com métodos padrão de Silva (1999).

Os dados foram submetidos à análise de variância com teste F significativo a $5 \%$. Posteriormente, as médias de cada tratamento foram analisadas pelo teste de ScottKnott (SCOTT \& KNOTT, 1974) a $5 \%$ de probabilidade, para comparação entre as médias.

\section{RESULTADOS E DISCUSSÃO}

\section{Análise do lixiviado}

Os tratamentos apresentaram lixiviação de nitrato segundo a seguinte ordem: $\mathrm{H} 1<\mathrm{H} 2<\mathrm{TA}$ (Tabela 2), embora esses resultados não tenham apresentado diferença estatística quando submetidos ao teste de Scott-Knott a 5 $\%$. Os valores obtidos neste estudo mostram que a utilização das dinamizações $\mathrm{CH} 3(\mathrm{H} 1)$ e $\mathrm{CH} 30(\mathrm{H} 2)$ da homeopatia Ammonium Carbonicum não contribuíram significativamente para a minimização da lixiviação de $\mathrm{NO}_{3}^{-}$. 
TABELA 2 - Quantidade de nitrogênio $\left(\mathrm{N}-\mathrm{NH}_{4}^{+} ; \mathrm{N}-\mathrm{NO}_{3}^{-} ; \mathrm{N}\right.$-mineral $)$ e volume de água lixiviados, em função dos tratamentos.

\begin{tabular}{lcccc}
\hline \multirow{2}{*}{ Tratamentos } & $\mathrm{N}-\mathrm{NH}_{4}^{+}$ & $\mathrm{N}-\mathrm{NO}_{3}^{-}$ & $\mathrm{N}$-mineral & Volume lixiviado \\
\cline { 2 - 4 } & & $\mathrm{kg} \mathrm{ha}^{-1}$ & & $\mathrm{~m}^{3} \mathrm{ha}^{-1}$ \\
\hline Ammonium Carbonicum $(\mathrm{CH} 3)$ & $4 \mathrm{a}^{*} \pm 1$ & $18 \mathrm{a} \pm 4$ & $21 \mathrm{a} \pm 4$ & $2.221 \mathrm{a} \pm 53$ \\
Ammonium Carbonicum $(\mathrm{CH} 30)$ & $4 \mathrm{a} \pm 1$ & $18 \mathrm{a} \pm 4$ & $21 \mathrm{a} \pm 4$ & $2.330 \mathrm{a} \pm 73$ \\
Testemunha & $4 \mathrm{a} \pm 2$ & $25 \mathrm{a} \pm 4$ & $28 \mathrm{a} \pm 4$ & $2.290 \mathrm{a} \pm 363$ \\
\hline
\end{tabular}

*Médias sucedidas por mesma letra minúscula na coluna não diferem significativamente pelo teste de Scott-Knott a 5\% de probabilidade.

Analisando os teores de $\mathrm{NO}_{3}^{-}$nos lixiviados coletados em cada tratamento, supõe-se que a lixiviação do $\mathrm{NO}_{3}^{-}$só possa ter sido oriunda da mineralização do $\mathrm{N}$ naturalmente existente no solo, uma vez que os tratamentos não receberam nenhuma adubação nitrogenada. Tendo em vista as diluições utilizadas no preparo das soluções homeopáticas, a quantidade de $\mathrm{N}$ oriunda do Ammonium Carbonicum é virtualmente nula. Anjos \& Mattiazzo (2000) também observaram lixiviação de $\mathrm{NO}_{3}^{-}$em Latossolo, com perdas de $\sim 103 \mathrm{mg} \mathrm{N}$ por vaso.

Na Figura 1, estão mostradas as concentrações de $\mathrm{NH}_{4}^{+}$e $\mathrm{NO}_{3}^{-}$obtidas nos lixiviados em função do volume de poros, em cada tratamento aplicado. Em todos os tratamentos houve um súbito aumento na concentração de $\mathrm{NH}_{4}^{+}$lixiviado, entre 0,6 e 0,9 volumes de poros. Esse aumento relativo de $\mathrm{NH}_{4}^{+}$no solo pode ser atribuído ao desenvolvimento qualitativo do tipo da microbiota do solo, favorecendo a mineralização de $\mathrm{NH}_{4}^{+}$(MARQUES et al., 2000), nos respectivos volumes citados.

Provavelmente causados também pelo aumento na atividade da microbiota, houveram vários picos nas concentrações de $\mathrm{NO}_{3}^{-}$. As concentrações de nitrato nos lixiviados por vezes foi superior (Figura 1) ao limite estabelecido pela Organização Mundial de Saúde, $10 \mathrm{mg}$ $\mathrm{N}-\mathrm{L}^{-1}$, que é também o limite adotado no Brasil (Portaria MS 518 de 25 de Março de 2004). A concentração de nitrato nos lixiviados dos tratamentos TA, H1 e H2 até 0,9 volume de poros e entre 1,5-1,6 (H2) e 1,6-1,7 (TA) volume de poros apresentou-se superior a $10 \mathrm{mg} \mathrm{N}-\mathrm{L}^{-1}$.

Ocorreram alguns picos na concentração de nitrato nos lixiviados. Essas altas concentrações caracterizam a baixa retenção desse íon no solo. Além disso, observouse que entre 1,5-1,8 volume de poros os tratamentos $\mathrm{H} 2 \mathrm{e}$ TA apresentaram dois picos de lixiviação de $\mathrm{NO}_{3}^{-}$; entretanto, o tratamento $\mathrm{H} 1$ não apresentou a mesma tendência. Tal observação é um indício de que o tratamento $\mathrm{H} 1$ poderia ter tido algum efeito no solo, minimizando a lixiviação de $\mathrm{NO}_{3}^{-}$. Contudo, essa tendência não apresentou diferença estatística e, portanto, essa menor lixiviação deve ser atribuída a variáveis não controladas no experimento.

\section{Análise do solo}

Algumas variáveis analisadas no solo, ao final do experimento, não foram influenciadas pelos os tratamentos, são elas: $\mathrm{NO}_{3}^{-} ; \mathrm{NH}_{4}^{+} ; \mathrm{pH} ; \mathrm{P} ; \mathrm{Mg} ; \mathrm{K} ; \mathrm{T} ; \mathrm{V}$; P-rem; $\mathrm{Zn}$; Fe; B e S. Por outro lado, os valores de $\mathrm{H}+\mathrm{Al}$; $\mathrm{Ca}$; C orgânico; $\mathrm{Mn}$ e $\mathrm{Cu}$ foram diferentes (Tabela 3).

Os teores de C orgânico no solo (Tabela 3), ao final do experimento, foram menores nos tratamentos homeopáticos $\mathrm{H} 1$ e H2, comparados ao tratamento TA. Esta diferença é atribuída à hipótese de que a homeopatia Ammonium Carbonicum provoque alguma alteração na microbiota do solo. Neste caso, possivelmente seu efeito está na aceleração da mineralização da matéria orgânica do solo e, conseqüentemente, na diminuição dos teores de $\mathrm{C}$ orgânico. Paralelamente, os tratamentos $\mathrm{H} 1$ e H2 apresentaram tendência à menor quantidade de $\mathrm{NO}_{3}^{-}$ lixiviado (Tabela 2) e maiores teores de $\mathrm{N}$ na parte aérea da planta no tratamento H2 (Tabela 4). Porém, comparando-se os valores de $\mathrm{C}$ orgânico observados ao final do experimento, em cada tratamento, com o valor no solo $\left(14,5 \mathrm{~g} \mathrm{~kg}^{-1}\right)$ no início do experimento, verifica-se uma variação muito pequena ou nula, a qual carece de significância prática .

A diferença encontrada nos valores de $\mathrm{H}+\mathrm{Al}$ (Tabela 3) nos tratamentos $\mathrm{H} 1 \mathrm{e} \mathrm{H}$, comparados ao valor observado no tratamento TA, possivelmente seja atribuída também à hipótese de que a homeopatia Ammonium Carbonicum teria efeito sob a microbiota do solo. Avaliando o fato de que a decomposição de organismos vivos e seus produtos metabólicos causam algumas transformações químicas, como a produção de $\mathrm{H}_{2} \mathrm{CO}_{3}$, com posterior liberação de íons $\mathrm{H}^{+}$resultantes da ionização de grupos $-\mathrm{COOH}\left(\mathrm{R}-\mathrm{COOH} \rightarrow \mathrm{R}-\mathrm{COO}^{-}+\mathrm{H}^{+}\right.$) (ANJOS et al., 
1999), provavelmente a liberação de $\mathrm{H}^{+}$resultante dos metabólitos reativos da microbiota do solo, nos tratamentos $\mathrm{H} 1$ e $\mathrm{H} 2$, passaria a ocupar os sítios de adsorção de cátions, que foram provavelmente extraídos pela cultura ou lixiviados. Porém, assim como foi citado anteriormente, ao comparar os valores de $\mathrm{H}+\mathrm{Al}$ no solo no

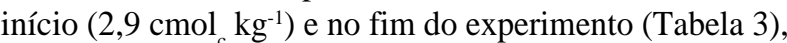
nota-se que, neste caso também, obteve-se uma variação muito pequena para ter alguma significância prática que comprove a ação do tratamento homeopático aplicado.

O menor teor de Ca observado no solo (Tabela 3) ao fim do experimento para o tratamento $\mathrm{H} 2$ pode ser atribuído à maior absorção desse elemento pela planta neste tratamento (Tabela 4), além de fornecer um indício de que o tratamento com a homeopatia Ammonium Carbonicum (CH30) poderia auxiliar a planta na absorção de $\mathrm{Ca}$. A variação obtida no teor de $\mathrm{Cu}$ no solo seguiu a mesma tendência do Ca para o H2, apresentou valor mais baixo que no tratamento TA e, concomitantemente, maior teor na parte aérea da planta. Os teores de Mn variaram de 22 a 28 $\mathrm{mg} \mathrm{kg}^{-1}$, indicando efeito dos tratamentos (Tabela 3). Estes valores são considerados altos (ALVAREZ et al., 1999), porém não seguem a mesma tendência nos teores foliares, como observado para $\mathrm{Ca}$ e $\mathrm{Cu}$.

Os altos teores de $\mathrm{N}$ mineral $\left(\mathrm{NO}_{3}^{-}+\mathrm{NH}_{4}^{+}\right)$no solo, após o término das lixiviações, ao final do experimento, indicam a capacidade de suprimento de $\mathrm{N}$ a partir da mineralização da matéria orgânica do solo. Porém, esses altos valores de $\mathrm{N}$ mineral remanescente no solo podem ser atribuídos ao fato das amostras terem sido secas previamente à determinação. Durante a secagem, provavelmente parte da matéria orgânica humificada do solo foi mineralizada, possivelmente, liberando $\mathrm{N}$ (SANGOI et al., 2003).

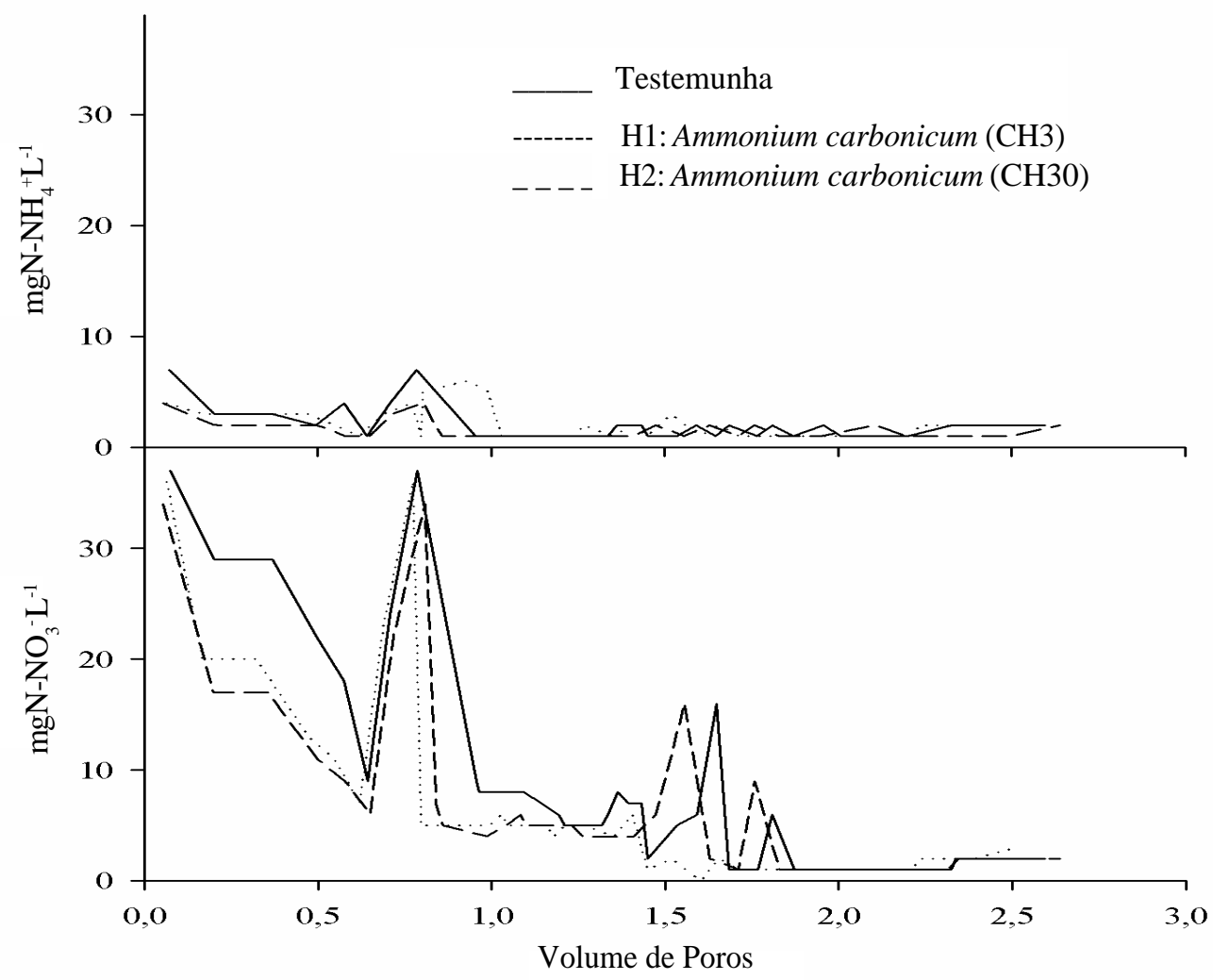

FIGURA 1 - Concentração de $\mathrm{N}-\mathrm{NH}_{4}^{+}$e $\mathrm{N}-\mathrm{NO}_{3}^{-}$nos lixiviados em função do volume de poros percolado em cada tratamento. 
TABELA 3 - Valores das características analisadas no solo, ao fim do experimento para cada tratamento.

\begin{tabular}{lccc}
\hline \multicolumn{1}{c}{ Variáveis } & $\mathbf{H 1}^{\mathbf{1}}$ & $\mathbf{H 2}^{\mathbf{2}}$ & $\mathbf{T A}^{\mathbf{3}}$ \\
\hline $\mathrm{NO}_{3}^{-}\left(\mathrm{mg} \mathrm{kg}^{-1}\right)$ & $113 \mathrm{a}^{*} \pm 6$ & $147^{\mathrm{a}} \pm 22$ & $151 \mathrm{a} \pm 27$ \\
$\mathrm{NH}_{4}^{+}\left(\mathrm{mg} \mathrm{kg}^{-1}\right)$ & $26 \mathrm{a} \pm 4$ & $21 \mathrm{a} \pm 1$ & $31 \mathrm{a} \pm 2$ \\
$\mathrm{H}+\mathrm{Al}\left(\mathrm{cmol}_{\mathrm{c} \mathrm{kg}} \pm \mathbf{2}\right)$ & $3,1 \mathrm{~b} \pm 0,0$ & $3,1 \mathrm{~b} \pm 0,2$ & $2,5 \mathrm{a} \pm 0,3$ \\
$\mathrm{Ca}\left(\mathrm{cmol}_{\mathrm{c} \mathrm{k}}{ }^{-1}\right)$ & $3,7 \mathrm{~b} \pm 0,2$ & $3,3 \mathrm{a} \pm 0,0$ & $3,7 \mathrm{~b} \pm 0,2$ \\
$\mathrm{C}$ orgânico $\left(\mathrm{g} \mathrm{kg}^{-1}\right)$ & $14,5 \mathrm{a} \pm 0,0$ & $14,1 \mathrm{a} \pm 0,3$ & $15,7 \mathrm{~b} \pm 0,7$ \\
$\mathrm{Mn}\left(\mathrm{mg} \mathrm{kg}^{-1}\right)$ & $28 \mathrm{c} \pm 0,8$ & $25 \mathrm{~b} \pm 1,7$ & $22 \mathrm{a} \pm 0,6$ \\
$\mathrm{Cu}\left(\mathrm{mg} \mathrm{kg}^{-1}\right)$ & $8,6 \mathrm{~b} \pm 0,1$ & $8,3 \mathrm{~b} \pm 0,4$ & $7,3 \mathrm{a} \pm 0,4$ \\
\hline
\end{tabular}

*Médias sucedidas por mesma letra minúscula na linha não diferem significativamente pelo teste de Scott-Knott a 5\% de probabilidade. ${ }^{1}$ Ammonium Carbonicum $(\mathrm{CH} 3) ;{ }^{2}$ Ammonium Carbonicum $(\mathrm{CH} 30) ;{ }^{3}$ Testemunha.

TABELA 4 - Médias das variáveis analisadas na matéria seca da parte aérea da planta, no final do experimento, para cada tratamento.

\begin{tabular}{lrrr}
\hline \multicolumn{1}{c}{ Variável } & \multicolumn{1}{c}{$\mathbf{H 1}^{\mathbf{1}}$} & \multicolumn{1}{c}{$\mathbf{H 2}^{\mathbf{2}}$} & $\mathbf{T A}^{\mathbf{3}}$ \\
\hline Matéria seca (g por vaso) & $2,66 \mathrm{a}^{*} \pm 0,3$ & $2,09 \mathrm{a} \pm 0,2$ & $1,43 \mathrm{a} \pm 0,1$ \\
$\mathrm{~N}\left(\mathrm{~g} \mathrm{~kg}^{-1}\right)$ & $8,4 \mathrm{a} \pm 0,4$ & $9,1 \mathrm{a} \pm 0,3$ & $8,8 \mathrm{a} \pm 1,0$ \\
$\mathrm{P}\left(\mathrm{g} \mathrm{kg}^{-1}\right)$ & $2,4 \mathrm{a} \pm 0,5$ & $2,4 \mathrm{a} \pm 0,1$ & $2,9 \mathrm{a} \pm 0,3$ \\
$\mathrm{~K}\left(\mathrm{~g} \mathrm{~kg}^{-1}\right)$ & $21 \mathrm{a} \pm 0,5$ & $19 \mathrm{a} \pm 0$ & $20 \mathrm{a} \pm 1$ \\
$\mathrm{Ca}\left(\mathrm{g} \mathrm{kg}^{-1}\right)$ & $4,7 \mathrm{a} \pm 0,2$ & $4,9 \mathrm{a} \pm 0,3$ & $4,4 \mathrm{a} \pm 0,3$ \\
$\mathrm{Mg}\left(\mathrm{g} \mathrm{kg}^{-1}\right)$ & $2,5 \mathrm{a} \pm 0,2$ & $2,4 \mathrm{a} \pm 0,2$ & $2,3 \mathrm{a} \pm 0,0$ \\
$\mathrm{~S}\left(\mathrm{~g} \mathrm{~kg}^{-1}\right)$ & $0,7 \mathrm{a} \pm 0,1$ & $0,9 \mathrm{a} \pm 0,0$ & $0,8 \mathrm{a} \pm 0,1$ \\
$\mathrm{~B}\left(\mathrm{mg} \mathrm{kg}^{-1}\right)$ & $14 \mathrm{a} \pm 2$ & $13 \mathrm{a} \pm 1$ & $18 \mathrm{a} \pm 2$ \\
$\mathrm{Cu}\left(\mathrm{mg} \mathrm{kg}^{-1}\right)$ & $2,3 \mathrm{a} \pm 0,2$ & $2,5 \mathrm{a} \pm 0,2$ & $3,2 \mathrm{~b} \pm 0,1$ \\
$\mathrm{Mn}\left(\mathrm{mg} \mathrm{kg}^{-1}\right)$ & $42 \mathrm{a} \pm 11$ & $40 \mathrm{a} \pm 6$ & $39 \mathrm{a} \pm 2$ \\
$\mathrm{Zn}\left(\mathrm{mg} \mathrm{kg}^{-1}\right)$ & $21 \mathrm{a} \pm 3,7$ & $18 \mathrm{a} \pm 0,4$ & $21 \mathrm{a} \pm 4,4$ \\
\hline
\end{tabular}

*Médias sucedidas por mesma letra minúscula na linha não diferem significativamente pelo teste de Scott-Knott a 5\% de probabilidade. ${ }^{1}$ Ammonium Carbonicum (CH3); ${ }^{2}$ Ammonium Carbonicum $(\mathrm{CH} 30) ;{ }^{3}$ Testemunha.

\section{Análise do solo em diferentes profundidades}

Os valores das variáveis analisadas nas diferentes profundidades estão apresentados na Tabela 5. As demais variáveis analisadas $\left(\mathrm{NO}_{3}^{-} ; \mathrm{NH}_{4}^{+} ; \mathrm{P} ; \mathrm{Ca} ; \mathrm{CTC} ; \mathrm{t}\right.$; C orgânico; Zn; B; e S) não sofreram efeito significativo dos tratamentos aplicados. Houve uma tendência, comum a todos os tratamentos, dos valores de $\mathrm{pH}$ serem mais baixos na profundidade $0-0,05 \mathrm{~m}$. Esse valor aumentou ligeiramente na profundidade subjacente. Esta variação no valor do $\mathrm{pH}$ em profundidade, somada à igual variação nos valores de $\mathrm{H}+\mathrm{Al}$, pode explicar os menores valores obtidos de P-remanescente nas camadas mais ácidas e menor disponibilidade de micronutrientes, $\mathrm{Mn}$ e $\mathrm{Cu}$, nessas mesmas profundidades.

Os teores de K (Tabela 5) tiveram aumento gradual em função das profundidades em todos os tratamentos. A lixiviação do K para as camadas mais profundas pode ser atribuída à menor energia de ligação que este íon apresenta com os minerais do solo, devido ao seu baixo potencial iônico, tornando-o mais propenso à lixiviação quando comparado aos outros cátions presentes no solo.

\section{Análise da parte aérea da planta}

Apenas o teor de $\mathrm{Cu}$ foi influenciado pelos tratamentos, quando submetidos ao teste de Scott-Knott a $5 \%$ (Tabela 4). A produção de MS obtida ao fim do experimento em cada tratamento foi semelhante à da testemunha. Lisboa (2004) realizou um ensaio em exatamente idênticas condições experimentais às deste trabalho. Ela obteve, no tratamento que recebeu adubação mineral completa, teores de $\mathrm{N}$ na matéria seca da parte aérea iguais a $8,1 \pm 1,5 \mathrm{~g} \mathrm{~kg}^{-1}$. Neste experimento, os teores de $\mathrm{N}$ na matéria seca da parte aérea foram semelhantes (Tabela 4) e até ligeiramente superiores aos de Lisboa (2004). 
TABELA 5 - Valores observados de pH, $\mathrm{K}, \mathrm{Mg}, \mathrm{H}+\mathrm{Al}$, P-remanescente, $\mathrm{Mn}$ e $\mathrm{Cu}$ ao término do experimento em diferentes profundidades de solo, para cada tratamento homeopático.

\begin{tabular}{|c|c|c|c|}
\hline \multirow{3}{*}{ Variável } & \multicolumn{3}{|c|}{ Profundidade } \\
\hline & \multicolumn{3}{|c|}{- Ammonium Carbonicum $(\mathrm{CH} 3)$} \\
\hline & $0-0,05 \mathrm{~m}$ & $0,05-0,10 \mathrm{~m}$ & $0,10-0,15 \mathrm{~m}$ \\
\hline $\mathrm{pH}\left(\mathrm{H}_{2} \mathrm{O}\right)$ & $6,1 a^{*} \pm 0,2$ & $6,4 a \pm 0$ & $6,3 a \pm 0,2$ \\
\hline $\mathrm{K}\left(\mathrm{mg} \mathrm{kg}^{-1}\right)$ & $41 \mathrm{a} \pm 5$ & $51 \mathrm{a} \pm 8$ & $75 b \pm 14$ \\
\hline $\operatorname{Mg}\left(\mathrm{cmol}_{\mathrm{c}} \mathrm{kg}^{-1}\right)$ & $0,6 \mathrm{a} \pm 0,1$ & $0,8 \mathrm{a} \pm 0,2$ & $0,6 \mathrm{a} \pm 0,1$ \\
\hline $\mathrm{H}+\mathrm{Al}\left(\mathrm{cmol}_{\mathrm{c}} \mathrm{kg}^{-1}\right)$ & $3,5 b \pm 0,2$ & $3,0 \mathrm{a} \pm 0,2$ & $2,9^{\mathrm{a}} \pm 0$ \\
\hline P-rem $\left(\mathrm{mg} \mathrm{kg}^{-1}\right)$ & $11,5 \mathrm{a} \pm 0,7$ & $14,0 \mathrm{~b} \pm 0,4$ & $15,0 \mathrm{~b} \pm 0,7$ \\
\hline $\operatorname{Mn}\left(\mathrm{mg} \mathrm{kg}^{-1}\right)$ & $30,1 b \pm 1,6$ & $30,1 b \pm 2,5$ & $24,4 \mathrm{a} \pm 0,8$ \\
\hline \multirow[t]{3}{*}{$\mathrm{Cu}\left(\mathrm{mg} \mathrm{kg}^{-1}\right)$} & $9,7 \mathrm{a} \pm 0,6$ & $8,6 a \pm 0,5$ & $7,6 \mathrm{a} \pm 0,1$ \\
\hline & \multicolumn{3}{|c|}{-Ammonium Carbonicum (CH 30)- } \\
\hline & $0-0,05 \mathrm{~m}$ & $0,05-0,10 \mathrm{~m}$ & $0,10-0,15 \mathrm{~m}$ \\
\hline $\mathrm{pH}\left(\mathrm{H}_{2} \mathrm{O}\right)$ & $6,1 a^{*} \pm 0,1$ & $6,2 \mathrm{a} \pm 0,2$ & $6,4 \mathrm{a} \pm 0,1$ \\
\hline $\mathrm{K}\left(\mathrm{mg} \mathrm{kg}^{-1}\right)$ & $33 a \pm 3$ & $45 \mathrm{a} \pm 3$ & $59 a \pm 9$ \\
\hline $\operatorname{Mg}\left(\mathrm{cmol}_{\mathrm{c}} \mathrm{kg}^{-1}\right)$ & $0,8 \mathrm{~b} \pm 0,2$ & $0,4 \mathrm{a} \pm 0,2$ & $0,7 b \pm 0,2$ \\
\hline $\mathrm{H}+\mathrm{Al}\left(\mathrm{cmol}_{\mathrm{c}} \mathrm{kg}^{-1}\right)$ & $3,9 b \pm 0,5$ & $2,9 \mathrm{a} \pm 0$ & $2,6 \mathrm{a} \pm 0$ \\
\hline P-rem $\left(\mathrm{mg} \mathrm{kg}^{-1}\right)$ & $11,8 \mathrm{a} \pm 0,7$ & $14,4 \mathrm{a} \pm 1,8$ & $17,4 \mathrm{a} \pm 0,8$ \\
\hline $\operatorname{Mn}\left(\mathrm{mg} \mathrm{kg}^{-1}\right)$ & $27,5 \mathrm{a} \pm 0,8$ & $25,9 a \pm 3$ & $22,7 \mathrm{a} \pm 3,6$ \\
\hline \multirow[t]{3}{*}{$\mathrm{Cu}\left(\mathrm{mg} \mathrm{kg}^{-1}\right)$} & $9,6 \mathrm{~b} \pm 0,5$ & $8,1 \mathrm{a} \pm 0,4$ & $7,2 \mathrm{a} \pm 0,9$ \\
\hline & \multicolumn{3}{|c|}{$\longrightarrow$ Testemunha- } \\
\hline & $0-0,05 \mathrm{~m}$ & $0,05-0,10 \mathrm{~m}$ & $0,10-0,15 \mathrm{~m}$ \\
\hline $\mathrm{pH}\left(\mathrm{H}_{2} \mathrm{O}\right)$ & $6,2 a^{*} \pm 0,2$ & $6,3 a \pm 0,2$ & $6,2 \mathrm{a} \pm 0,2$ \\
\hline $\mathrm{K}\left(\mathrm{mg} \mathrm{kg}^{-1}\right)$ & $37 a \pm 6$ & $49 a \pm 16$ & $65 a \pm 13$ \\
\hline $\operatorname{Mg}\left(\mathrm{cmol}_{\mathrm{c}} \mathrm{kg}^{-1}\right)$ & $0,8 \mathrm{a} \pm 0,2$ & $0,7 \mathrm{a} \pm 0,2$ & $0,5 \mathrm{a} \pm 0$ \\
\hline $\mathrm{H}+\mathrm{Al}\left(\mathrm{cmol}_{\mathrm{c}} \mathrm{kg}^{-1}\right)$ & $2,8 \mathrm{a} \pm 0,2$ & $2,6 a \pm 0$ & $2,6 a \pm 0$ \\
\hline P-rem $\left(\mathrm{mg} \mathrm{kg}^{-1}\right)$ & $12,5 a \pm 1$ & $14,4 b \pm 0,6$ & $15,1 b \pm 0$ \\
\hline $\operatorname{Mn}\left(\mathrm{mg} \mathrm{kg}^{-1}\right)$ & $21,9 a \pm 0,6$ & $22,1 \mathrm{a} \pm 1,4$ & $20,8 \mathrm{a} \pm 0,1$ \\
\hline $\mathrm{Cu}\left(\mathrm{mg} \mathrm{kg}^{-1}\right)$ & $7,9 \mathrm{c} \pm 0,3$ & $7,3 b \pm 0,3$ & $6,9 a \pm 0,3$ \\
\hline
\end{tabular}

*Médias sucedidas por mesma letra minúscula na linha não diferem significativamente pelo teste de Scott-Knott a 5\% de probabilidade.

No entanto, as quantidades de $\mathrm{N}$ acumuladas na planta, em função da matéria seca produzida, foram largamente diferentes. O tratamento com adubação mineral completa de Lisboa (2004) acumulou 0,21 g de $\mathrm{N}$ por vaso, contrastando com apenas $0,02-0,01 \mathrm{~g}$ de $\mathrm{N}$ por vaso para $\mathrm{H} 1, \mathrm{H} 2$ e TA. Os resultados de matéria seca do presente experimento podem, portanto, ser atribuídos à baixa capacidade de suprimento de $\mathrm{N}$ às plantas, quando o mesmo é oriundo apenas da mineralização da matéria orgânica do solo, sem adubação nitrogenada alguma.

O baixo desenvolvimento vegetativo obtido neste experimento, comparado com a matéria seca obtida no tratamento com adubação mineral completa de Lisboa (2004), 25,4 \pm 3 g por vaso, pode ser atribuído, pelo menos em parte, à indisponibilidade de $\mathrm{N}$ às plantas. Devido ao efeito concentração, causado pelo baixo crescimento, os teores de P e K na planta (Tabela 4) foram muito superiores aos teores obtidos para estes nutrientes no tratamento com adubação mineral completa de Lisboa (2004) (1,2 \pm 0,1 $\mathrm{mg} \mathrm{P} \mathrm{kg}{ }^{-1}$ e $\left.10 \pm 2 \mathrm{mg} \mathrm{K} \mathrm{kg}^{-1}\right)$. Além disso, $\mathrm{P}$ e $\mathrm{K}$ encontravamse no solo (Tabela 1) em concentrações consideradas "médias" (ALVAREZ et al., 1999). Portanto, esses dois nutrientes não devem ter sido tão limitantes ao crescimento quanto N. Contudo, os teores dos demais nutrientes analisados apresentaram valores variáveis, com algumas deficiências, como observado para $\mathrm{S}$; $\mathrm{B} ; \mathrm{Mg}$ e $\mathrm{Cu}$, apesar de se apresentarem em níveis "médios a altos" no solo (ALVAREZ et al., 1999) no início do experimento.

\section{CONCLUSÃO}

Este estudo mostrou o efeito da homeopatia Ammonium Carbonicum nas dinamizações CH3 e CH30 
sobre a mineralização da matéria orgânica, evidenciado pela diminuição no teor de C orgânico do solo. Entretanto, este efeito foi pequeno e não acompanhado pelas demais variáveis analisadas. Portanto, neste estudo não foi comprovado o efeito da homeopatia Ammonium Carbonicum, nas dinamizações CH3 e CH30, no sistema solo.

\section{REFERÊNCIAS BIBLIOGRÁFICAS}

ALVAREZ, V. H.; NOVAIS, R. F.; BARROS, N. F.; CANTARUTTI, R. B.; LOPES, A. S. Interpretação dos resultados das análises de solos. In: RIBEIRO, A. C.; GUIMARÃES, P. T. G.; ALVAREZ, V. H. (Eds.). Recomendação para o uso de corretivos e fertilizantes em Minas Gerais: $5^{\text {a }}$ aproximação. Viçosa: UFV, 1999. cap. 5, p. 25-32.

ALVAREZ, V. H.; RIBEIRO, A. C. Calagem. In: RIBEIRO, A. C.; GUIMARÃES, P. T. G.; ALVAREZ, V. H. (Eds.). Recomendação para o uso de corretivos e fertilizantes em Minas Gerais: $5^{\text {a }}$ aproximação. Viçosa: UFV, 1999. cap. 8, p. 43-60.

ANDRADE, F. M. C.; CASALI, V. W. D.; DEVITA, B.; CECON, P. R.; BARBOSA, L. C. A. Efeito de homeopatias no crescimento e na produção de cumarina em chambá (Justicia pectoralis Jacq.). Revista Brasileira de Plantas Medicinais, Botucatu, v. 4, n. 1, p. 19-28, out. 2001.

ANJOS, A. R. M.; MATTIAZZO, M. E. Lixiviação de íons inorgânicos em solos repetidamente tratados com biossólidos. Revista Brasileira de Ciência do Solo, Viçosa, v. 24, n. 4, p. 927-938, out./dez. 2000.

ANJOS, L. H. C.; PEREIRA, M. G.; RAMOS, D. P. Matéria orgânica e pedogênese. In: SANTOS, G. A.; CAMARGO, F. A. O. Fundamentos da matéria orgânica do solo: ecossistemas tropicais \& subtropicais. Porto Alegre: Gênesis, 1999. cap. 6, p. 91-116.

BETTI, L.; BORGHINI, F.; NANI, D. Plant models for fundamental research in homeopathy. Homeopathy, London, v. 92, p. 129-130, 2003.

BRASIL. Instrução Normativa n 7, de 17 de maio de 1999. Dispõe sobre normas para a produção de produtos orgânicos vegetais e animais. Diário Oficial [da] República Federativa do Brasil, Brasília, v. 99, n. 94, p. 11-14, 19 maio 1999. Seção 1.
BREMNER, J. M. Inorganic forms of nitrogen. In: BLACK, C. A. (Ed.). Methods of soil analysis: part 2. Madison: ASA, 1965. (Agron. Monogr., 9).

CARVALHO, L. M.; CASALI, V. W. D.; CECON, P. R.; SOUZA, M. A.; LISBOA, S. P. Efeito de potências decimais da homeopatia de Arnica montana sobre plantas de artemísia. Revista Brasileira de Plantas Medicinais, Botucatu, v. 6, n. 1, p. 46-50, out. 2003.

CARVALHO, L. M.; CASALI, V. W. D.; LISBOA, S. P.; SOUZA, M. A. Efeito da homeopatia na recuperação de plantas de artemísia [Tanacetum parthenicum (L.) SchultzBip] submetidas à deficiência hídrica. Revista Brasileira de Plantas Medicinais, Botucatu, v. 6, n. 2, p. 20-27, fev. 2004.

CASTRO, D. M. Preparações homeopáticas em plantas de cenoura, beterraba, capim-limão e chambá. 2002. 227 f. Tese (Doutorado em Fitotecnia) - Universidade Federal de Viçosa, Viçosa, 2002.

ELMI, A. A. A.; MADRAMOOTOO, C.; EGEH, M.; LIU, A.; HAMEL, C. Environmental and agronomic implications of water table and nitrogen fertilization management. Journal of Environmental Quality, Madison, v. 31, n. 6, p. 1858-1867, Nov./Dec. 2002.

EMPRESA BRASILEIRA DE PESQUISA AGROPECUÁRIA. Manual de métodos de análise de solo. 2. ed. Rio de Janeiro, 1997. 212 p.

HAMMAN, B.; KONING, G.; LOK, K. H. Homeopathically prepared gibberellic acid and barley seed germination. Homeopathy, London, v. 92, p. 140-144, 2003.

LATHOUD, J. A. Matéria médica homeopática. São Paulo: Robe, 2002.

LISBOA, C. C. Nitrogênio e adubação orgânica: lixiviação, efeito homeopático, mineralização e métodos de determinação de nitrato. 2004. 142 p. Dissertação (Mestrado) - Universidade Federal de Lavras, Lavras, 2004.

MARQUES, T. C. L. L.; VASCONCELOS, C. A.; PEREIRA FILHO, I.; FRANÇA, G. E.; CRUZ, J. C. Envolvimento de dióxido de carbono e mineralização de nitrogênio em Latossolo Vermelho-Amarelo com diferentes manejos. Pesquisa Agropecuária Brasileira, Brasília, v. 35, n. 3, p. 581-589, maio 2000. 
PANG, X. P.; LETEY, J. Organic farming: challenge of timing nitrogen availability to crop nitrogen requirements. Soil Science Society of America Journal, Madison, v. 64, n. 1, p. 247-253, Jan./Feb. 2000.

REY, L. Thermoluminescence of ultra-high dilutions of lithium chloride and sodium chloride. Physica A, Amsterdam, v. 323, p. 67-74, May. 2003.

SANGOI, L.; ERNANI, P. R.; LECH, V. A.; RAMPAZZO, C. Lixiviação de nitrogênio afetada pela forma de aplicação da uréia e manejo dos restos culturais de aveia em dois solos com texturas contrastante. Ciência Rural, Santa Maria, v. 33, n. 1, p. 65-70, jan./fev. 2003.

SCOTT, A. J.; KNOTT, M. Accouter analysis methods for grouping means in the analysis of variants. Biometrics, Saint Louis, v. 30, n. 3, p. 507-512, Sept. 1974.

SILVA, F. C. Manual de análises químicas de solos, plantas e fertilizantes. Brasília, DF: Embrapa, 1999. 370 p. 